\title{
A geometric perspective on lifting
}

\author{
Michele Conforti \\ Università di Padova, conforti@math.unipd.it \\ Gérard Cornuéjols * \\ Carnegie Mellon University and Université d'Aix-Marseille, gc0v@andrew.cmu.edu \\ Giacomo Zambelli \\ Università di Padova, giacomo@math.unipd.it
}

May 2009, revised December 2009

\begin{abstract}
Recently, it has been shown that minimal inequalities for a continuous relaxation of mixed integer linear programs are associated with maximal lattice-free convex sets. In this paper, we show how to lift these inequalities for integral nonbasic variables by considering maximal lattice-free convex sets in a higher-dimensional space. We apply this approach to several examples. In particular, we identify cases where the lifting is unique.
\end{abstract}

\section{Introduction}

A classical topic in integer programming is that of lifting, introduced by Gomory [13] in the context of the group problem, and further elaborated by Padberg [16]: given mixed integer sets $Q \subset \mathbb{R}^{n}$ and $R \subset \mathbb{R}^{n+p}$ such that $Q$ is the restriction of $R$ obtained by setting the last $p$ variables to 0 , and given a valid inequality $\sum_{i=1}^{n} a_{j} x_{j} \leq b$ for $Q$, find coefficients $a_{n+1}, \ldots, a_{n+p}$ such that $\sum_{i=1}^{n+p} a_{j} x_{j} \leq b$ is valid for $R$. Current state-of-the-art integer programming solvers routinely use lifted knapsack covers, lifted flow covers and other liftings. The lifting coefficients $a_{n+1}, \ldots, a_{n+p}$ may be computed sequentially, choosing the best possible value at each step. However, different orderings of the variables usually lead to different answers. An aspect of liftings that has received attention is that of sequence-independent lifting (Wolsey [17], Gu, Nemhauser, Savelsberg [14]). In this paper, we revisit liftings from a geometric perspective, building on recent work relating minimal inequalities to maximal lattice-free convex sets. Our results are best described in the context of an infinite model which we present next.

Let $S$ be the set of integral points in some rational polyhedron in $\mathbb{R}^{n}$ such that $\operatorname{dim}(\operatorname{conv}(S))=$ $n$ (for example $S$ could be the set of nonnegative integral points), and let $f \in \operatorname{conv}(S) \backslash \mathbb{Z}^{n}$.

\footnotetext{
${ }^{*}$ Supported by NSF grant CMMI0653419, ONR grant N00014-03-1-0188 and ANR grant BLAN06-1-138894.
} 
We consider the following semi-infinite model.

$$
\begin{aligned}
x= & f+\sum_{r \in \mathbb{R}^{n}} r s_{r}+\sum_{r \in \mathbb{R}^{n}} r y_{r} \\
& x \in S \\
& s_{r} \geq 0, \quad r \in \mathbb{R}^{n} \\
& y_{r} \geq 0, y_{r} \in \mathbb{Z}, \quad r \in \mathbb{R}^{n} \\
& s, y \text { have finite support. }
\end{aligned}
$$

The infinite vectors $s$ and $y$ having finite support means that they are nonzero only in a finite number of entries. Model (1) is a natural abstraction of the simplex tableau. Indeed, setting all but a finite number of the $s_{r}$ and $y_{r}$ variables to zero reduces (1) to a problem in tableau form with right-hand-side $f$, where $x$ are the basic variables, and the $s_{r}$ and $y_{r}$ variables not set to zero are the nonbasic ones. Since $S$ is a set of integral point in a polyhedron, the condition $x \in S$ enforces the integrality of the basic variables. When $S=\mathbb{Z}^{n}$, model (1) is the infinite group problem of Gomory.

Given two functions $\psi$ and $\pi$ from $\mathbb{R}^{n}$ to $\mathbb{R}$, the inequality

$$
\sum_{r \in \mathbb{R}^{n}} \psi(r) s_{r}+\sum_{r \in \mathbb{R}^{n}} \pi(r) y_{r} \geq 1
$$

is valid for (1) if it holds for every $(x, s, y)$ satisfying (1). If (2) is valid, we say that the function $(\psi, \pi)$ is valid for $(1)$. A valid function $(\psi, \pi)$ is minimal if there is no valid function $\left(\psi^{\prime}, \pi^{\prime}\right)$ distinct from $(\psi, \pi)$ such that $\psi^{\prime}(r) \leq \psi(r), \pi^{\prime}(r) \leq \pi(r)$ for all $r \in \mathbb{R}^{n}$. Note that, since all components of $s$ and $y$ are nonnegative, one is only interested in studying minimal valid functions.

Information about valid inequalities for (1) automatically transfers to the problem of cutting-off a fractional basic solution of the linear programming relaxation. Most cutting planes used in practice (Gomory mixed integer cuts, Mixed Integer Rounding inequalities, knapsack covers, flow covers, lift-and-project cuts and many other) are valid for Gomory's corner polyhedron, which is the convex hull of solutions to (1) where $S=\mathbb{Z}^{n}$ and all but a finite number of the variables $s_{r}$ and $y_{r}$ are set to 0 .

One of the most effective classes of cutting planes used in solvers is that of Gomory Mixed Integer cuts, which correspond to valid functions for (1) when $n=1$ and $S=\mathbb{Z}$. It is well known that, among all cutting planes derived from a single equation, Gomory Mixed Integer cuts have the best possible coefficients (i.e. the smallest) on the nonbasic continuous variables. To transfer this notion to the general setting of (1), Dey and Wolsey [11] proposed to study the following simpler model, where the integer variables $y_{r}$ are all set to zero.

$$
\begin{aligned}
x= & f+\sum_{r \in \mathbb{R}^{n}} r s_{r} \\
& x \in S \\
& s_{r} \geq 0, \quad r \in \mathbb{R}^{n} \\
& s \text { has finite support. }
\end{aligned}
$$


We refer to this model as the continuous semi-infinite relaxation relative to $f$. Given a valid function $\psi$ for (3), the function $\pi$ is a lifting of $\psi$ if $(\psi, \pi)$ is valid for (1). If $\psi$ is a minimal valid function for (3) and $\pi$ is a lifting of $\psi$ such that $(\psi, \pi)$ is minimal, we say that $\pi$ is a minimal lifting of $\psi$.

We remark that, given any valid function $\psi$ for (3) and a lifting $\pi$ of $\psi$, the function $\pi^{\prime}$ defined by $\pi^{\prime}(r)=\min \{\psi(r), \pi(r)\}$ is also a lifting of $\psi$. Indeed, given $(\bar{s}, \bar{y})$ satisfying (1), we show that $\sum_{r \in \mathbb{R}^{n}} \psi(r) \bar{s}_{r}+\sum_{r \in \mathbb{R}^{n}} \pi^{\prime}(r) \bar{y}_{r} \geq 1$. Let $(\tilde{s}, \tilde{y})$ be defined by $\tilde{s}_{r}=\bar{s}_{r}, \tilde{y}_{r}=\bar{y}_{r}$ for every $r \in \mathbb{R}^{n}$ such that $\pi(r) \leq \psi(r)$, and $\tilde{s}_{r}=\bar{s}_{r}+\bar{y}_{r}, \tilde{y}_{r}=0$ for every $r \in \mathbb{R}^{n}$ such that $\psi(r)<$ $\pi(r)$. One can readily verify that $(\tilde{s}, \tilde{y})$ satisfies $(1)$, hence $\sum_{r \in \mathbb{R}^{n}} \psi(r) \tilde{s}_{r}+\sum_{r \in \mathbb{R}^{n}} \pi(r) \tilde{y}_{r} \geq 1$. Furthermore, $\sum_{r \in \mathbb{R}^{n}} \psi(r) \bar{s}_{r}+\sum_{r \in \mathbb{R}^{n}} \pi^{\prime}(r) \bar{y}_{r}=\sum_{r \in \mathbb{R}^{n}} \psi(r) \tilde{s}_{r}+\sum_{r \in \mathbb{R}^{n}} \pi(r) \tilde{y}_{r} \geq 1$

In particular, if $\psi$ is a minimal valid function for (3) and $\pi$ is a minimal lifting of $\psi$, then $\pi \leq \psi$.

We first concentrate on deriving the best possible lifting coefficient of one single integer variable. Namely, given $d \in \mathbb{R}^{n}$, we consider the model

$$
\begin{aligned}
x= & f+\sum_{r \in \mathbb{R}^{n}} r s_{r}+d z \\
& x \in S \\
& s_{r} \geq 0, \quad r \in \mathbb{R}^{n} \\
& z \geq 0, z \in \mathbb{Z}, \quad \\
& s \text { has finite support. }
\end{aligned}
$$

Given a minimal valid function $\psi$ for $(3)$, let $\pi_{\ell}(d)$ be the minimum scalar $\lambda$ such that the inequality

$$
\sum_{r \in \mathbb{R}^{n}} \psi(r) s_{r}+\lambda z \geq 1
$$

is valid for (4).

Note that, if $\pi$ is a lifting of $\psi$, then $\sum_{r \in \mathbb{R}^{n}} \psi(r) s_{r}+\pi(d) z \geq 1$ is valid for (4). Thus, by definition of $\pi_{\ell}$, we have that $\pi_{\ell} \leq \pi$ for every lifting $\pi$ of $\psi$. In general, the function $\left(\psi, \pi_{\ell}\right)$ is not valid for $(1)$. However, when $\left(\psi, \pi_{\ell}\right)$ is valid, $\pi_{\ell}$ can be viewed as a trivial sequence-independent lifting of $\psi$ :

Proposition 1. Let $\psi$ be a minimal valid function for $(3)$. When $\left(\psi, \pi_{\ell}\right)$ is valid for $(1), \pi_{\ell}$ is the unique minimal lifting of $\psi$.

In this paper we give a geometric characterization of the function $\pi_{\ell}$, and use this characterization to analyze specific minimal valid functions $\psi$ for which $\pi_{\ell}$ is the unique minimal lifting.

A valid function $(\psi, \pi)$ is extreme for (1) if there do not exist distinct valid functions $\left(\psi^{1}, \pi^{1}\right),\left(\psi^{2}, \pi^{2}\right)$ such that $(\psi, \pi)=\frac{1}{2}\left(\psi^{1}, \pi^{1}\right)+\frac{1}{2}\left(\psi^{2}, \pi^{2}\right)$. Note that if $\psi$ is extreme for $(3)$, then $\psi$ is minimal.

Remark 2. If $\psi$ is extreme for (3) and $\left(\psi, \pi_{\ell}\right)$ is valid for $(1)$, then $\left(\psi, \pi_{\ell}\right)$ is extreme for (1).

Indeed, given valid functions $\left(\psi^{1}, \pi^{1}\right),\left(\psi^{2}, \pi^{2}\right)$ such that $(\psi, \pi)=\frac{1}{2}\left(\psi^{1}, \pi^{1}\right)+\frac{1}{2}\left(\psi^{2}, \pi^{2}\right)$, then $\psi_{1}=\psi_{2}=\psi$, since $\psi$ is extreme for (3), and $\pi_{1}=\pi_{2}=\pi_{\ell}$ since $\pi_{1} \geq \pi_{\ell}$ and $\pi_{2} \geq \pi_{\ell}$. 


\section{Lifting and $S$-free convex sets}

Minimal valid inequalities for (3) are well understood in terms of maximal $S$-free convex sets. We are interested in exploiting such characterization to provide a geometric interpretation of minimal liftings.

We observe that (4) is equivalent to the following

$$
\begin{aligned}
\left(\begin{array}{c}
x \\
x_{n+1}
\end{array}\right)= & \left(\begin{array}{l}
f \\
0
\end{array}\right)+\sum_{r \in \mathbb{R}^{n}}\left(\begin{array}{l}
r \\
0
\end{array}\right) s_{r}+\left(\begin{array}{l}
d \\
1
\end{array}\right) z \\
& \left(x, x_{n+1}\right) \in S \times \mathbb{Z}_{+} \\
& s_{r} \geq 0, \quad r \in \mathbb{R}^{n} \\
& z \geq 0, \\
& s \text { has finite support. }
\end{aligned}
$$

Indeed $(x, s, z)$ is a solution for (4) if and only if $\left(x, x_{n+1}, s, z\right)$ is a solution to (5) by setting $x_{n+1}=z$. Note that the above is obtained from the continuous semi-infinite relaxation relative to $\left(\begin{array}{l}f \\ 0\end{array}\right)$ by setting to 0 all variables relative to rays with nonzero $(n+1)$-th component, except for $\left(\begin{array}{l}d \\ 1\end{array}\right)$. Therefore, given any valid function $\bar{\psi}$ for the continuous semi-infinite relaxation relative to $\left(\begin{array}{l}f \\ 0\end{array}\right)$, then if we let $\psi(r)=\bar{\psi}\left(\begin{array}{l}r \\ 0\end{array}\right)$ for $r \in \mathbb{R}^{n}$ and $\lambda=\bar{\psi}\left(\begin{array}{l}d \\ 1\end{array}\right)$, the inequality $\sum_{r \in \mathbb{R}^{n}} \psi(r) s_{r}+\lambda z \geq 1$ is valid for (5) and for (4).

A convex set is $S$-free if it does not contain any point of $S$ in its interior. Maximal $S$-free convex sets were characterized in [6], where it was also shown that there is a one-to-one correspondence between minimal valid functions for (3) and maximal $S$-free convex sets with $f$ in their interior.

Theorem 3. [6] A full-dimensional convex set $B$ is a maximal $S$-free convex set if and only if it is a polyhedron such that $B$ does not contain any point of $S$ in its interior and each facet of $B$ contains a point of $S$ in its relative interior. Furthermore if $B \cap \operatorname{conv}(S)$ has nonempty interior, $\operatorname{lin}(B)$ contains $\operatorname{rec}(B \cap \operatorname{conv}(S))$.

We explain how minimal valid inequalities for (3) arise from maximal $S$-free convex sets. Let $B$ a polyhedron with $f$ in its interior, and let $a_{1}, \ldots, a_{t} \in \mathbb{R}^{q}$ such that $B=\{x \in$ $\left.\mathbb{R}^{n} \mid a_{i}(x-f) \leq 1, i=1 \ldots, t\right\}$. We define the function $\psi_{B}: \mathbb{R}^{n} \rightarrow \mathbb{R}$ by

$$
\psi_{B}(r)=\max _{i=1, \ldots, t} a_{i} r
$$

Note that the function $\psi_{B}$ is convex, subadditive, i.e. $\psi_{B}(r)+\psi_{B}\left(r^{\prime}\right) \geq \psi_{B}\left(r+r^{\prime}\right)$, and positively homogeneous, i.e. $\psi_{B}(\lambda r)=\lambda \psi_{B}(r)$ for every $\lambda \geq 0$.

We claim that, if $B$ is a maximal $S$-free convex set, then

$$
\sum_{r \in \mathbb{R}^{n}} \psi_{B}(r) s_{r} \geq 1 \quad \text { is valid for }(3) .
$$

Indeed, let $(x, s)$ be a solution of (3). Note that $x \in S$, thus $x$ is not in the interior of $B$. Then

$$
\sum_{r \in \mathbb{R}^{n}} \psi_{B}(r) s_{r}=\sum_{r \in \mathbb{R}^{n}} \psi_{B}\left(r s_{r}\right) \geq \psi_{B}\left(\sum_{r \in \mathbb{R}^{n}} r s_{r}\right)=\psi_{B}(x-f) \geq 1,
$$


where the first equation follows from positive homogeneity, the first inequality follows from subadditivity of $\psi_{B}$ and the last one follows from the fact that $x$ is not in the interior of $B$.

The above functions are minimal [6],[11]. It was proved in [6] that the converse is also true, namely that every minimal function valid for (3) is of the form $\psi_{B}$ where $B$ is a maximal $S$-free convex set with $f$ in its interior.

Example. We consider problem (1) when $n=1,0<f<1$ and $S=\mathbb{Z}$. In this case the only maximal $S$-free convex set containing $f$ is the interval $B=[0,1]$. Thus $B=\{x \in$ $\left.\mathbb{R} \mid-f^{-1}(x-f) \leq 1,(1-f)^{-1}(x-f) \leq 1\right\}$ and $\psi_{B}(r)=\max \left\{-f^{-1} r,(1-f)^{-1} r\right\}$.

Let $\psi$ be a minimal valid function for (3), and let $B=\left\{x \in \mathbb{R}^{n} \mid a_{i}(x-f) \leq 1, i=1, \ldots, t\right\}$ be a maximal $S$-free convex set with $f$ in its interior such that $\psi=\psi_{B}$. We define the set $B(\lambda) \subset \mathbb{R}^{n+1}$ as follows

$$
B(\lambda)=\left\{\left(x, x_{n+1}\right) \in \mathbb{R}^{n+1} \mid a_{i}(x-f)+\left(\lambda-a_{i} d\right) x_{n+1} \leq 1, i=1, \ldots, t\right\} .
$$

Theorem 4. The inequality $\sum_{r \in \mathbb{R}^{n}} \psi(r) s_{r}+\lambda z \geq 1$ is valid for (4) if and only if $B(\lambda)$ is $\left(S \times \mathbb{Z}_{+}\right)$-free.

Proof. Let $\bar{\psi}=\psi_{B(\lambda)}$. By construction, $\bar{\psi}\left(\begin{array}{l}r \\ 0\end{array}\right)=\psi(r)$ for all $r \in \mathbb{R}^{n}$, while $\bar{\psi}\left(\begin{array}{l}d \\ 1\end{array}\right)=\lambda$.

We show the "if" part of the statement. Given $\lambda$ such that $B(\lambda)$ is $\left(S \times \mathbb{Z}_{+}\right)$-free, it follows by claim (6) that the function $\bar{\psi}$ is valid for the continuous semi-infinite relaxation relative to $\left(\begin{array}{l}f \\ 0\end{array}\right)$. This implies that $\sum_{r \in \mathbb{R}^{n}} \psi(r) s_{r}+\lambda z \geq 1$ is valid for (4).

We now prove the "only if" part. Let $\lambda$ be such that $\sum_{r \in \mathbb{R}^{n}} \psi(r) s_{r}+\lambda z \geq 1$ is valid for (4). Given a point $\left(\begin{array}{c}\bar{x} \\ \bar{x}_{n+1}\end{array}\right) \in S \times \mathbb{Z}_{+}$, we show that such point is not in the interior of $B(\lambda)$. Indeed, let $\bar{r}=\bar{x}-\bar{x}_{n+1} d-f, \bar{z}=\bar{x}_{n+1}$, and $\left(\bar{s}_{r}\right)_{r \in \mathbb{R}^{n}}$ be defined by

$$
\bar{s}_{r}= \begin{cases}1 & \text { if } r=\bar{r} \\ 0 & \text { otherwise }\end{cases}
$$

Note that $f+\sum_{r \in \mathbb{R}^{n}} r \bar{s}_{r}+d \bar{z}=f+\bar{r}+\bar{x}_{n+1} d=\bar{x}$. Since $\bar{x} \in S$ and $\sum_{r \in \mathbb{R}^{n}} \psi(r) s_{r}+\lambda z \geq 1$ is valid for (4), we have

$$
\begin{aligned}
1 & \leq \sum_{r \in \mathbb{R}^{n}} \psi(r) \bar{s}_{r}+\lambda \bar{z}=\psi(\bar{r})+\lambda \bar{x}_{n+1}=\max _{i=1, \ldots, t} a_{i} \bar{r}+\lambda \bar{x}_{n+1} \\
& =\max _{i=1, \ldots, t}\left[a_{i}(\bar{x}-f)+\left(\lambda-a_{i} d\right) \bar{x}_{n+1}\right] .
\end{aligned}
$$

Thus there exists $i \in\{1, \ldots, t\}$ such that $a_{i}(\bar{x}-f)+\left(\lambda-a_{i} d\right) \bar{x}_{n+1} \geq 1$. This shows that $\left(\begin{array}{c}\bar{x} \\ \bar{x}_{n+1}\end{array}\right)$ is not in the interior of $B(\lambda)$.

Theorem 4 implies that $\pi_{\ell}(d)$ is the minimum value of $\lambda$ such that $B(\lambda)$ is $\left(S \times \mathbb{Z}_{+}\right)$-free.

Example (continued). In the previous example, let $d \in \mathbb{R}$ and $\lambda \in \mathbb{R}$. If $\lambda \neq 0$, then the set $B(\lambda)$ is the 2-dimensional polyhedron with two facets, containing the points $\left(\begin{array}{l}0 \\ 0\end{array}\right)$ and $\left(\begin{array}{l}1 \\ 0\end{array}\right)$ respectively and with one vertex, namely $\left(\begin{array}{l}f \\ 0\end{array}\right)+\lambda^{-1}\left(\begin{array}{l}d \\ 1\end{array}\right)$. If $\lambda=0$, then $B(\lambda)$ is the split set $[0,1]+\left\langle\left(\begin{array}{l}d \\ 1\end{array}\right)\right\rangle$. It is immediate to verify that, for $\lambda<0$, the interior of $B(\lambda)$ contains one of 
the integral points $\left(\begin{array}{c}\lfloor d\rfloor \\ 1\end{array}\right)$ or $\left(\begin{array}{c}\lceil d\rceil \\ 1\end{array}\right)$.

For example, let $f=\frac{1}{4}$. For $d=\frac{3}{2}, \psi_{B}(d)=2$. One can readily verify that $B(\lambda)$ is $\mathbb{Z} \times \mathbb{Z}_{+}$-free if and only if $\lambda \geq \frac{2}{3}$, otherwise it contains the point $\left(\begin{array}{l}2 \\ 1\end{array}\right)$. Hence $\pi_{\ell}(d)=\frac{2}{3}$.

For $d=1, \psi_{B}(d)=\frac{4}{3}$. It is immediate that $B(\lambda)$ is $\mathbb{Z} \times \mathbb{Z}_{+}$-free if and only if $\lambda \geq 0$, hence $\pi_{\ell}(d)=0$.
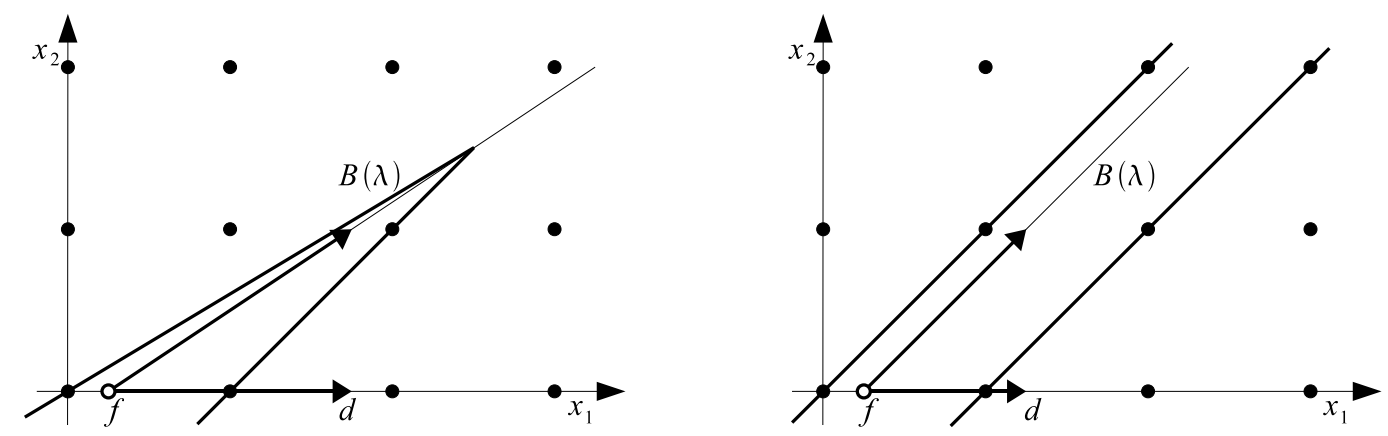

Figure 1: Example: $f=\frac{1}{4}$. Left: $d=\frac{3}{2}$. Right: $d=1$.

Theorem 5. Let $\psi$ be a minimal valid function for (3) and $\pi$ be a minimal lifting of $\psi$. Then there exists $\varepsilon>0$ such that $\psi, \pi$ and $\pi_{\ell}$ coincide on the ball of radius $\varepsilon$ centered at the origin.

Proof. Since $\psi$ is a minimal valid function for (3), there exists a maximal $S$-free convex set $B=\left\{x \in \mathbb{R}^{n} \mid a_{i}(x-f) \leq 1, i=1, \ldots, t\right\}$ such that $\psi=\psi_{B}$.

Let

$$
\alpha=\max _{1 \leq i, j \leq t} \max _{\|r\|=1}\left(a_{i}-a_{j}\right) r
$$

Since $B$ is a maximal $S$-free convex set, every facet of $B$ contains a point of $S$ in its relative interior. Hence, for $i=1, \ldots, t$, there exists $x^{i} \in S$ such that $a_{i}\left(x^{i}-f\right)=1$ and $a_{j}\left(x^{i}-f\right) \leq 1-\gamma_{i}, j \neq i$, for some positive $\gamma_{i}$. Let $\varepsilon>0$ such that $\varepsilon \alpha \leq \gamma_{i}$ for $i=1, \ldots, t$.

Let $d \in \mathbb{R}^{n}$ such that $\|d\| \leq \varepsilon$. We will show that, for every $\lambda<\psi(d), B(\lambda)$ contains a point of $S \times \mathbb{Z}_{+}$in its interior. By Theorem 4, this implies that $\pi_{\ell}(d) \geq \psi(d)$. Since $\pi_{\ell} \leq \pi \leq \psi$, this implies $\pi_{\ell}(d)=\pi(d)=\psi(d)$.

Let $i, 1 \leq i \leq t$, such that $\psi(d)=a_{i} d$. Let $\lambda=\psi(d)-\delta$ for some $\delta>0$. We show that $B(\lambda)$ contains the point $\left(\begin{array}{c}x^{i} \\ 1\end{array}\right)$ in its interior. Indeed, by $(7), B(\lambda)$ is the set of points in $\mathbb{R}^{n+1}$ satisfying the inequalities

$$
a_{j}(x-f)+\left[\left(a_{i}-a_{j}\right) d-\delta\right] x_{n+1} \leq 1, \quad j=1, \ldots, t .
$$

Substituting $\left(\begin{array}{c}x^{i} \\ 1\end{array}\right)$ we obtain

$$
\begin{aligned}
a_{i}\left(x^{i}-f\right) & -\delta \\
a_{j}\left(x^{i}-f\right)+\left(a_{i}-a_{j}\right) d-\delta & <1, \quad j=1, \ldots, t, j \neq i
\end{aligned}
$$


where the first inequality follows from $a_{i}\left(x^{i}-f\right)=1$, while the second follows from $a_{j}\left(x^{i}-f\right) \leq$ $1-\gamma_{i},\|d\| \leq \varepsilon$, and $\left(a_{i}-a_{j}\right)(d /\|d\|) \leq \alpha$ by our choice of $\alpha$.

Thus $\left(\begin{array}{c}x^{i} \\ 1\end{array}\right)$ is in the interior of $B(\lambda)$.

Example (continued). From the previous example where $n=1,0<f<1$ and $S=\mathbb{Z}$, note that $\pi_{\ell}(d)=\psi_{B}(d)$ for every $d \in[-f, 1-f]$. Indeed, if $d<0$, then $B(\lambda)$ contains $\left(\begin{array}{l}0 \\ 1\end{array}\right)$ for all $\lambda<\psi_{B}(d)$, while if $d \geq 0$ then $B(\lambda)$ contains $\left(\begin{array}{l}1 \\ 1\end{array}\right)$ for all $\lambda<\psi_{B}(d)$. Furthermore, for $\lambda=\psi_{B}(d)$, if $d<0$ the facet of $B(\lambda)$ containing $\left(\begin{array}{l}0 \\ 0\end{array}\right)$ is vertical and contains the point $\left(\begin{array}{l}0 \\ 1\end{array}\right)$, if $d \geq 0$ then the facet of $B(\lambda)$ containing $\left(\begin{array}{l}1 \\ 0\end{array}\right)$ is vertical and contains the point $\left(\begin{array}{l}1 \\ 1\end{array}\right)$.

Theorem 5 implies that, for every minimal valid function $\psi$ for (3), there exists a region $R_{\psi} \subseteq \mathbb{R}^{n}$ containing the origin in its interior such that $\psi$ and $\pi$ coincide in $R_{\psi}$ for every minimal lifting $\pi$ of $\psi$ for (1). Since $\psi$ is piecewise linear, it follows that $\pi$ is piecewise linear around the origin. This is in contrast with extreme functions $\pi$ for the pure integer semiinfinite relaxation (i.e. the set (1) where all the $s_{r}$ are set to 0 ) which need not be piecewise linear [4].

Lemma 6. Let $\psi$ be a minimal valid function, and $\pi$ be a minimal lifting of $\psi$. Then

i) For every $r \in \mathbb{R}^{n}$ and $w \in \mathbb{Z}^{n} \cap \operatorname{lin}(\operatorname{conv}(S))$, $\pi(r)=\pi(r+w)$.

ii) For every $r \in \mathbb{R}^{n}$ such that $r+w \in R_{\psi}$ for some $w \in \mathbb{Z}^{n} \cap \operatorname{lin}(\operatorname{conv}(S)), \pi(r)=\psi(r+w)$.

Proof. $i)$ Let $\bar{r} \in \mathbb{R}^{n}$ and $w \in \mathbb{Z}^{n} \cap \operatorname{lin}(\operatorname{conv}(S))$. Suppose $\pi(\bar{r}) \neq \pi(\bar{r}+w)$. Since $-w \in$ $\mathbb{Z}^{n} \cap \operatorname{lin}(\operatorname{conv}(S))$, we may assume $\pi(\bar{r})>\pi(\bar{r}+w)$. Since $w \in \mathbb{Z}^{n} \cap \operatorname{lin}(\operatorname{conv}(S))$, then a point $x \in \mathbb{R}^{n}$ is in $S$ if and only if $x+w \in S$. Thus a point $(\bar{x}, \bar{s}, \bar{y})$ satisfies (1) if and only if $\left(\bar{x}+w \bar{y}_{\bar{r}}, \bar{s}, \tilde{y}\right)$ satisfies (1), where $\tilde{y}_{\bar{r}}=0, \tilde{y}_{\bar{r}+w}=\bar{y}_{\bar{r}+w}+\bar{y}_{\bar{r}}$, and $\tilde{y}_{r}=\bar{y}_{r}$ for every $r \in \mathbb{R}^{n} \backslash\{\bar{r}, \bar{r}+w\}$. This shows that the function $\pi^{\prime}$ defined by $\pi^{\prime}(\bar{r})=\pi(\bar{r}+w), \pi^{\prime}(r)=\pi(r)$ for every $r \in \mathbb{R}^{n} \backslash\{\bar{r}\}$ is a lifting of $\psi$, contradicting the minimality of $\pi$.

ii) It follows from $i$ ) that $\pi(r)=\pi(r+w)$. By definition of $R_{\psi}, \pi(r+w)=\psi(r+w)$.

This lemma is closely related to a result of Balas and Jeroslow [3]. It implies the following property.

Theorem 7. If for every $r \in \mathbb{R}^{n}$ there exists $w^{r} \in \mathbb{Z}^{n} \cap \operatorname{lin}(\operatorname{conv}(S))$ such that $r+w \in R_{\psi}$, then there exists a unique minimal lifting of $\psi$, namely the function $\pi$ defined by $\pi(r)=\psi\left(r+w^{r}\right)$. Furthermore $\pi=\pi_{\ell}$.

Note that, if for some $r \in R_{\psi}$ there exists $w \in \mathbb{Z}^{n} \cap \operatorname{lin}(\operatorname{conv}(S))$ such that $r+w \in R_{\psi}$, then $\psi(r+w)=\psi(r)$.

Example (continued). From the previous example where $n=1,0<f<1$ and $S=\mathbb{Z}$, we have shown that $\psi(r)=\pi_{\ell}(r)$ for every $r \in[-f, 1-f]$. Note that, for every $r \in \mathbb{R}$, $r-\lfloor r+f\rfloor \in[-f, 1-f]$. Thus $\pi_{\ell}(r)=\psi(r-\lfloor r+f\rfloor)$ for all $r \in \mathbb{R}$, and $\pi_{\ell}$ is the unique minimal lifting of $\psi$. Thus $\pi_{\ell}(r)=\max \left\{-f^{-1}(r-\lfloor r+f\rfloor),(1-f)^{-1}(r-\lfloor r+f\rfloor)\right\}$. More explicitly, if $r-\lfloor r\rfloor<1-f$, then $\pi_{\ell}(r)=\frac{r-\lfloor r\rfloor}{1-f}$, while if $r-\lfloor r\rfloor \geq 1-f, \pi_{\ell}(r)=\frac{\lceil r\rceil-r}{f}$. 
Given a tableau row $x=f+\sum_{i=1}^{h} p^{i} s_{i}+\sum_{j=1}^{k} q^{j} y_{j}$, where $s_{i} \geq 0, i=1, \ldots, h$, and $y_{j} \geq 0$ and integer, $j=1, \ldots, h$, the inequality $\sum_{i=1}^{h} \psi\left(p^{i}\right) s_{i}+\sum_{j=1}^{k} \pi_{\ell}\left(q^{j}\right) y_{j} \geq 1$ is

$$
\sum_{\substack{i=1 \\ p^{i} \geq 0}}^{h} \frac{p^{i}}{1-f} s_{i}+\sum_{\substack{i=1 \\ p^{i}<0}}^{h}-\frac{p^{i}}{f} s_{i}+\sum_{\substack{j=1 \\ q^{j}-\left\lfloor q^{j}\right\rfloor<1-f}}^{k} \frac{q^{j}-\left\lfloor q^{j}\right\rfloor}{1-f} y_{j}+\sum_{\substack{j=1 \\ q^{j}-\left\lfloor q^{j}\right\rfloor \geq 1-f}}^{k} \frac{\left\lceil q^{j}\right\rceil-q^{j}}{f} y_{j} \geq 1
$$

which is the Gomory Mixed Integer Cut associated with the tableau row.

\section{Applications}

We illustrated in Section 2 how our geometric approach can be used to derive Gomory's mixed integer cuts. In this section, we give three examples of how it can be applied to the multi-row case.

\subsection{Wedge inequalities}

We consider the problem (1) where $n=2$ and $S=\mathbb{Z} \times \mathbb{Z}_{+}$. We focus on inequalities arising from maximal $S$-free convex sets with 2 sides and one vertex. We call such sets wedges.
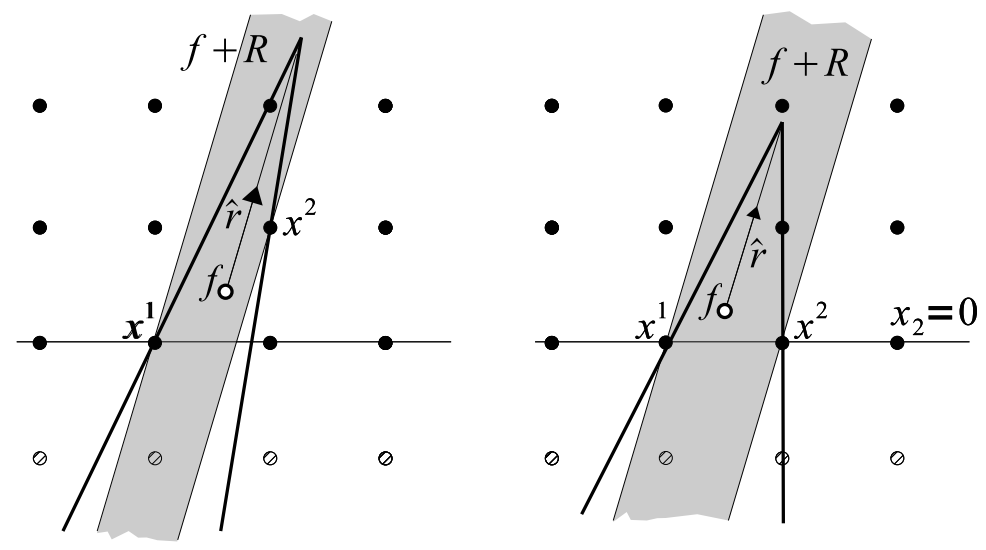

Figure 2: Wedges and corresponding region $R+\{f\}$ shaded in gray. The inequality corresponding to the wedge on the right has a unique minimal lifting.

Let $B=\left\{x \in \mathbb{R}^{2} \mid a_{i}(x-f) \leq 1, i=1,2\right\}$ be such a maximal $S$-free convex set. Since $B$ is $S$-free, its only vertex must be in the interior of $\operatorname{conv}(S), \operatorname{rec}(B)$ has dimension 2 and for every nonzero element $r \in \operatorname{rec}(B), r_{2}<0$.

Note that $\operatorname{rec}(\operatorname{conv}(S))=\mathbb{R} \times \mathbb{R}_{+}$and $B$ has empty lineality space. By Theorem 3 , $\operatorname{lin}(B) \supseteq \operatorname{rec}(B \cap \operatorname{conv}(S))$, hence $\operatorname{rec}(B) \cap \operatorname{conv}(S)=\emptyset$. In particular, $(\mathbb{R} \times\{0\}) \cap \operatorname{rec}(B)=\emptyset$, thus by symmetry we may assume $a_{1}\left(\begin{array}{l}1 \\ 0\end{array}\right)<0$ and $a_{2}\left(\begin{array}{l}1 \\ 0\end{array}\right)>0$, that is $a_{11}<0$ and $a_{21}>0$.

Let $\hat{r}$ be a nonzero vector such that $a_{1} \hat{r}=a_{2} \hat{r}$. Clearly the second coordinate of $\hat{r}$ is nonzero. Note that any point $x \in \mathbb{R}^{2}$ can be uniquely written as $x=f+\alpha^{x} \hat{r}+\beta^{x}\left(\begin{array}{l}1 \\ 0\end{array}\right)$ where $\alpha^{x}, \beta^{x} \in \mathbb{R}$. Let $\bar{x} \in S$ be a point in the relative interior of one of the two facets of $B$, say 
$a_{h}(\bar{x}-f)=1, a_{k}(\bar{x}-f)<1$. Note that $0>\left(a_{k}-a_{h}\right)(\bar{x}-f)=\beta^{\bar{x}}\left(a_{k 1}-a_{h 1}\right)$, hence $\beta^{\bar{x}}<0$ if $h=1$ and $\beta^{\bar{x}}>0$ if $h=2$. Let $x^{1}$ be a point of $S$ in the relative interior of the facet defined by $a_{1}(x-f) \leq 1$ such that $\beta^{x^{1}}$ is largest possible, and $x^{2}$ be a point of $S$ in the relative interior of the facet defined by $a_{2}(x-f) \leq 1$ such that $\beta^{x^{2}}$ is smallest possible. Let $\beta_{i}=\beta^{x^{i}}$. Note that $\beta_{1}<0<\beta_{2}$. We define the region $R=\left[\beta_{1}, \beta_{2}\right]+\langle\hat{r}\rangle$. (See Figure 2.)

Lemma 8. For every $d \in R, \pi_{\ell}(d)=\psi_{B}(d)$.

Proof. Let $d \in R$, that is $d=\alpha \hat{r}+\beta\left(\begin{array}{l}1 \\ 0\end{array}\right)$, for some $\alpha \in \mathbb{R}$ and $\beta \in\left[\beta_{1}, \beta_{2}\right]$. We consider the case $\beta \leq 0$. The case $\beta \geq 0$ is similar.

Note that $\left(a_{1}-a_{2}\right) d=\alpha\left(a_{1}-a_{2}\right) \hat{r}+\beta\left(a_{11}-a_{21}\right) \geq 0$ since $\left(a_{1}-a_{2}\right) \hat{r}=0, \beta \leq 0, a_{11}<0$ and $a_{21}>0$. Hence $\psi_{B}(d)=\max \left\{a_{1} d, a_{2} d\right\}=a_{1} d$.

We will show that, for every $\lambda<\psi_{B}(d)$, the set $B(\lambda)$ defined in (7) contains the point $\left(\begin{array}{c}x^{1} \\ 1\end{array}\right)$ in its interior. By Theorem 4, this will imply $\pi_{\ell}(d) \geq \psi_{B}(d)$, and thus $\pi_{\ell}(d)=\psi_{B}(d)$.

Let $\lambda=\psi_{B}(d)-\delta$ for some $\delta>0$. Then $B(\lambda)$ is the set of $x \in \mathbb{R}^{3}$ satisfying

$$
\begin{aligned}
a_{1}(x-f) & -\delta x_{3} \leq 1 \\
a_{2}(x-f)+\left(a_{1}-a_{2}\right) d x_{3}-\delta x_{3} & \leq 1 .
\end{aligned}
$$

Substituting $\left(\begin{array}{c}x^{1} \\ 1\end{array}\right)$ in the first inequality, we obtain $a_{1}\left(x^{1}-f\right)-\delta=1-\delta<1$. Substituting in the second inequality, we obtain

$$
\begin{aligned}
a_{2}\left(x^{1}-f\right)+\left(a_{1}-a_{2}\right) d-\delta & =\alpha^{x^{1}} a_{2} \hat{r}+\beta_{1} a_{21}+\alpha\left(a_{1}-a_{2}\right) \hat{r}+\beta\left(a_{11}-a_{21}\right)-\delta \\
& =\alpha^{x^{1}} a_{1} \hat{r}+\beta_{1} a_{11}+\left(\beta-\beta_{1}\right)\left(a_{11}-a_{21}\right)-\delta \\
& \leq a_{1}\left(x^{1}-f\right)-\delta=1-\delta<1
\end{aligned}
$$

where the first inequality in the last row follows from $\beta_{1} \leq \beta, a_{11}<0, a_{21}>0$. Thus $\left(\begin{array}{c}x^{1} \\ 1\end{array}\right)$ is in the interior of $B(\lambda)$.

Let $y^{1}$ and $y^{2}$ be the intersection of the facets defined by $a_{1}(x-f) \leq 1$ and $a_{2}(x-f) \leq 1$, respectively, with the axis $x_{2}=0$. That is $a_{1}\left(y^{1}-f\right)=1, y_{2}^{1}=0$, and $a_{2}\left(y^{2}-f\right)=1$, $y_{2}^{2}=0$. Since $B$ is $S$-free, $y_{1}^{2}-y_{1}^{1} \leq 1$, where equality holds if and only if $y^{1}, y^{2}$ are integral. Furthermore, it is not difficult to show that $\beta_{2}-\beta_{1} \leq y_{1}^{2}-y_{1}^{1}$. Thus $\beta_{2}-\beta_{1}=1$ if and only if $y^{1}, y^{2}$ are integral vectors. In this case, for every $r \in \mathbb{R}^{2}$ there exists $w^{r} \in \mathbb{Z} \times\{0\}$ such that $r+w^{r} \in R$. Since $\operatorname{lin}(\operatorname{conv}(S))=\mathbb{R} \times\{0\}$, by Theorem $7, \pi_{\ell}(r)$ is the unique minimal lifting of $\psi_{B}$, and $\pi_{\ell}(r)=\psi_{B}\left(r+w^{r}\right)$ for every $r \in \mathbb{R}^{2}$.

Dey and Wolsey [11] show that $\psi_{B}$ is extreme for (3) if and only if $B$ contains at least three points of $S$. Thus Remark 2 implies the following:

Theorem 9. If $B$ contains at least three points of $S$ and $B \cap(\mathbb{R} \times\{0\})$ is an interval of length one, then $\left(\psi_{B}, \pi_{\ell}\right)$ is an extreme inequality for (1).

Example. Let $f=\left(\begin{array}{c}\frac{2}{3} \\ \frac{1}{3}\end{array}\right)$ and $S=\mathbb{Z} \times \mathbb{Z}_{+}$. Consider the wedge

$$
W=\left\{x \in \mathbb{R}^{2} \mid-3\left(x_{1}-\frac{2}{3}\right)+3\left(x_{2}-\frac{1}{3}\right) \leq 1, \frac{12}{5}\left(x_{1}-\frac{2}{3}\right)-\frac{3}{5}\left(x_{2}-\frac{1}{3}\right) \leq 1\right\} .
$$




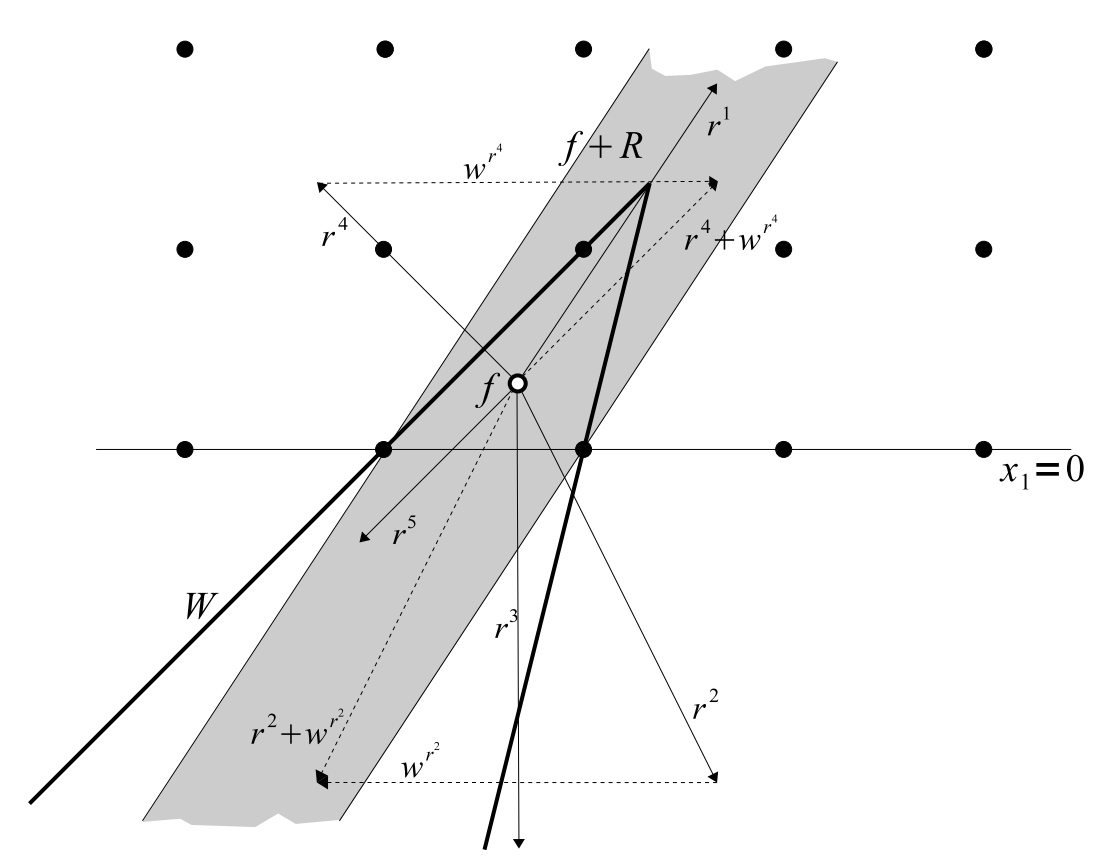

Figure 3: Set $W$ in the example and corresponding region $R+\{f\}$ shaded in gray.

The set $W$ is a maximal $S$-free convex set, as one may easily see from Figure 3 .

The corresponding minimal function is given by

$$
\psi(r)=\max \left\{-3 r_{1}+3 r_{2}, \frac{12}{5} r_{1}-\frac{3}{5} r_{2}\right\}
$$

One can easily verify that the vector $\hat{r}=\left(\begin{array}{l}2 \\ 3\end{array}\right)$ satisfies $-3 \hat{r}_{1}+3 \hat{r}_{2}=\frac{12}{5} \hat{r}_{1}-\frac{3}{5} \hat{r}_{2}$, and that the region $R$ is thus given by $R=\left[-\frac{4}{9}, \frac{5}{9}\right]+\left(\begin{array}{l}2 \\ 3\end{array}\right)$. This can be written as

$$
R=\left\{r \in \mathbb{R}^{2} \mid-\frac{4}{9} \leq r_{1}-\frac{2}{3} r_{2} \leq \frac{5}{9}\right\}
$$

For every $r \in R^{2}$, define the integral vector $w^{r}$ by $w_{1}^{r}=-\left\lfloor r_{1}-\frac{2}{3} r_{2}+\frac{4}{9}\right\rfloor, w_{2}^{r}=0$. Note that $w^{r} \in \operatorname{lin}(S) \cap \mathbb{Z}^{2}$ and $r+w^{r} \in R$ for all $r \in \mathbb{R}^{2}$. The unique minimal lifting for $\psi$ is therefore the function $\pi$ defined by $\pi(r)=\psi\left(r+w^{r}\right)$. The explicit formula is given by

$$
\pi(r)=\max \left\{-3\left(r_{1}-\left\lfloor r_{1}-\frac{2}{3} r_{2}+\frac{4}{9}\right\rfloor\right)+3 r_{2}, \frac{12}{5}\left(r_{1}-\left\lfloor r_{1}-\frac{2}{3} r_{2}+\frac{4}{9}\right\rfloor\right)-\frac{3}{5} r_{2}\right\} .
$$

Suppose now we are given the following two rows of the optimal simplex tableau for the linear relaxation of a mixed integer program.

$$
\begin{gathered}
x_{1}=\frac{2}{3}+\quad x_{3}+\quad x_{4} \quad-\quad x_{6}-\frac{4}{5} x_{7} \\
x_{2}=\frac{1}{3}+\frac{3}{2} x_{3}-2 x_{4}-\frac{7}{3} x_{5}+x_{6}-\frac{4}{5} x_{7} \\
x_{1}, x_{2}, x_{3}, x_{4}, x_{5}, x_{6}, x_{7} \geq 0 \\
x_{1}, x_{2}, x_{4}, x_{6} \in \mathbb{Z}
\end{gathered}
$$


The lifted inequality determined by the wedge $W$ is $\psi\left(r^{1}\right) x_{3}+\pi\left(r^{2}\right) x_{4}+\psi\left(r^{3}\right) x_{5}+\pi\left(r^{4}\right) x_{6}+$ $\psi\left(r^{5}\right) x_{7} \geq 1$, where $r^{1}=\left(\begin{array}{c}1 \\ \frac{3}{2}\end{array}\right), r^{2}=\left(\begin{array}{c}1 \\ -2\end{array}\right), r^{3}=\left(\begin{array}{c}0 \\ -\frac{7}{3}\end{array}\right), r^{4}=\left(\begin{array}{c}-1 \\ 1\end{array}\right), r^{5}=\left(\begin{array}{c}-\frac{4}{5} \\ -\frac{4}{5}\end{array}\right)$. This gives the inequality

$$
\frac{3}{2} x_{3}-\frac{6}{5} x_{4}+\frac{7}{5} x_{5}+\frac{9}{5} x_{6} \geq 1
$$

Note that the non-lifted inequality (that is, the inequality obtained from $W$ if we ignored the integrality conditions on $x_{4}$ and $x_{6}$ ) is

$$
\frac{3}{2} x_{3}+\frac{18}{5} x_{4}+\frac{7}{5} x_{5}+6 x_{6} \geq 1
$$

\subsection{Simplicial polytopes}

In this section we focus on valid inequalities for (3) arising from maximal lattice-free simplicial polytopes, in the case where $S=\mathbb{Z}^{n}$. Recall that a polytope is simplicial if each of its facets is a simplex.

Let $B=\left\{x \in \mathbb{R}^{n} \mid a_{i}(x-f) \leq 1, i=1, \ldots, t\right\}$ be an $n$-dimensional maximal lattice-free simplicial polytope and let $v^{1}, \ldots, v^{p}$ be its vertices. For $i=1, \ldots, t$, let $V_{i} \subset\{1, \ldots, p\}$ be the set of indices of vertices of the facet defined by $a_{i}(x-f) \leq 1$, that is $a_{i}\left(v^{j}-f\right)=1$ for all $j \in V_{i}$. Let $r^{i}=v^{i}-f, i=1, \ldots, p$. Note that, since $B$ is simplicial, $\left\{r^{j} \mid j \in V_{i}\right\}$ consists of $n$ linearly independent vectors, for $i=1, \ldots, t$, and $a_{i} r^{j}=1$ for all $j \in V_{i}$, while $a_{i} r^{j}<1$ for all $j \notin V_{i}$.

Let $\bar{x}$ be an integral point in the relative interior of the facet defined by $a_{i}(x-f) \leq 1$, that is $a_{i}(\bar{x}-f)=1, a_{j}(\bar{x}-f)<1, j \neq i$. Then $\bar{x}$ can be uniquely written as $\bar{x}=f+\sum_{j \in V_{i}} \bar{\alpha}_{j} r^{j}$, where $\sum_{j \in V_{i}} \bar{\alpha}_{j}=1, \bar{\alpha}_{j} \geq 0, j \in V_{i}$. Let $R(\bar{x})=\left\{\sum_{j \in V_{i}} \alpha_{j}{ }^{j} \mid 0 \leq \alpha_{j} \leq \bar{\alpha}_{j}, j \in V_{i}\right\}$.

Let us denote by $\mathcal{I}$ the set of all points $\bar{x}$ in $\mathbb{Z}^{n}$ such that $\bar{x}$ is contained in the relative interior of some facet of $B$. Let $R=\cup_{\bar{x} \in \mathcal{I}} R(\bar{x})$.

Lemma 10. For every $d \in R, \pi_{\ell}(d)=\psi_{B}(d)$.

Proof. We only need to show that, given $\bar{x} \in \mathcal{I}$ and $d \in R(\bar{x}), \pi_{\ell}(d)=\psi_{B}(d)$. By symmetry we may assume that $\bar{x}$ is in the relative interior of the facet defined by $a_{1}(\bar{x}-f) \leq 1$, and that $V_{1}=\{1, \ldots, n\}$. Let $\bar{\alpha}_{1}, \ldots, \bar{\alpha}_{n}$ nonnegative such that $\sum_{j=1}^{n} \bar{\alpha}_{j}=1$ and $\bar{x}=f+\sum_{j=1}^{n} \bar{\alpha}_{j} r^{j}$. Since $d \in R(\bar{x})$, there exist $\alpha_{1}, \ldots, \alpha_{n}$ such that $d=\sum_{j=1}^{n} \alpha_{j} r^{j}$ and $0 \leq \alpha_{j} \leq \bar{\alpha}_{j}, j=1, \ldots, n$. Note that, for $i=1, \ldots, t,\left(a_{1}-a_{i}\right) d=\sum_{j=1}^{n} \alpha_{j}\left(a_{1}-a_{i}\right) r^{j} \geq 0$. Thus $\psi_{B}(d)=a_{1} d$.

We will show that, for every $\lambda<\psi_{B}(d)$, the set $B(\lambda)$ defined as in (7) contains the point $\left(\begin{array}{l}\bar{x} \\ 1\end{array}\right)$ in its interior. By Theorem 4, this will imply $\pi_{\ell}(d) \geq \psi_{B}(d)$, and thus $\pi_{\ell}(d)=\psi_{B}(d)$.

Let $\lambda=\psi_{B}(d)-\delta$ for some $\delta>0$. Then $B(\lambda)$ is the set of $x \in \mathbb{R}^{n+1}$ satisfying

$$
\begin{aligned}
& a_{1}(x-f) \quad-\delta x_{n+1} \leq 1, \\
& a_{i}(x-f)+\left(a_{1}-a_{i}\right) d x_{n+1}-\delta x_{n+1} \leq 1, \quad i=2, \ldots, t .
\end{aligned}
$$

Substituting $\left(\begin{array}{l}\bar{x} \\ 1\end{array}\right)$ in the first inequality, we obtain $a_{1}(\bar{x}-f)-\delta=1-\delta<1$. Substituting 
in the $i$ th inequality, $i=2, \ldots, n+1$, we obtain

$$
\begin{aligned}
a_{i}(\bar{x}-f)+\left(a_{1}-a_{i}\right) d-\delta & =\sum_{j=1}^{n} \bar{\alpha}_{j} a_{i} r^{j}+\sum_{j=1}^{n} \alpha_{j}\left(a_{1}-a_{i}\right) r^{j}-\delta \\
& =\sum_{j=1}^{n} \bar{\alpha}_{j}-\sum_{j=1}^{n} \bar{\alpha}_{j}\left(1-a_{i} r^{j}\right)+\sum_{j=1}^{n} \alpha_{j}\left(1-a_{i} r^{j}\right)-\delta \\
& =1-\sum_{j=1}^{n}\left(\bar{\alpha}_{j}-\alpha_{j}\right)\left(1-a_{i} r^{j}\right)-\delta \\
& \leq 1-\delta<1
\end{aligned}
$$

where the equality in the second line follows from $a_{i} r^{j}=1$ for $j=1, \ldots, n$, the equality on the third line follows from $\sum_{j=1}^{n} \bar{\alpha}_{j}=1$, while the first inequality on the last line follows from $\alpha_{j} \leq \bar{\alpha}_{j}$ and $a_{i} r^{j} \leq 1$.

In light of Theorem 7, we are interested in cases where for every $r \in \mathbb{R}^{n}$ there exists $w^{r} \in \mathbb{Z}^{n}$ such that $r+w^{r} \in R$, since in this case $\pi_{\ell}$ is the unique minimal lifting.

Dey and Wolsey [10] studied the case $n=2$. In this case maximal lattice free polytopes are either triangles or quadrilaterals [15]. Dey and Wolsey show that the above property holds if and only if $B$ is a triangle containing at least four integral points (see Figure 4), while it does not hold if $B$ is a triangle containing exactly three integral points or if $B$ is a quadrilateral. They also show that, when $B$ is a triangle with at least four integral points, $\left(\psi_{B}, \pi_{\ell}\right)$ is extreme for $(1)$. This fact also follows from Remark 2 and from the fact that $\psi_{B}$ is extreme for (3) whenever $B$ is a maximal lattice-free triangle [9].
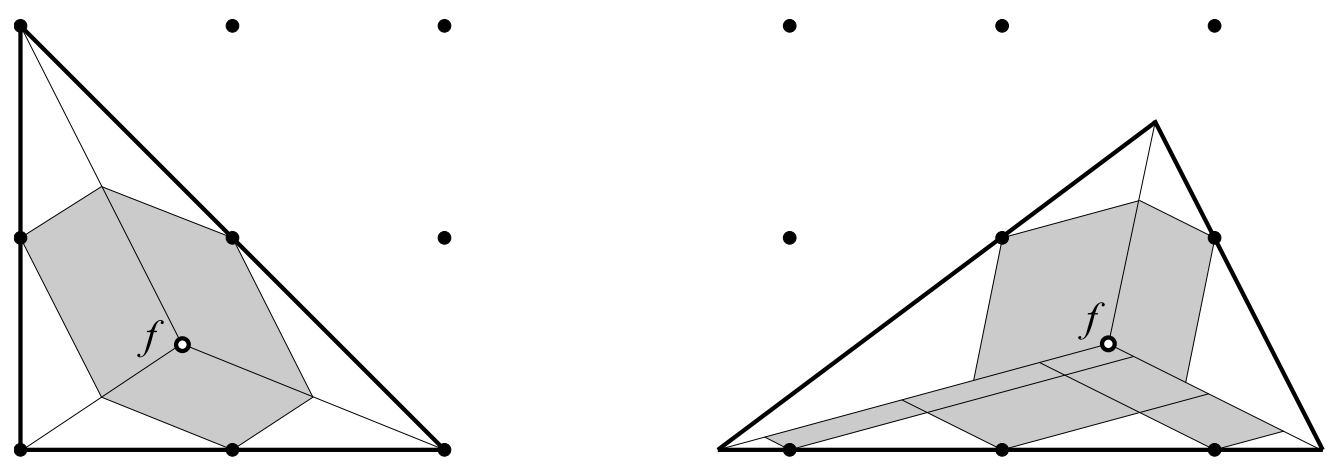

Figure 4: Lattice free triangles giving inequalities with a unique minimal lifting. Region $R+\{f\}$ is shaded.

We next show that the above property holds when $B$ is the $n$-dimensional simplex $\operatorname{conv}\left\{0, n e_{1}, \ldots, n e_{n}\right\}$, where $e_{i}$ denotes the $i$ th unit vector. We assume that $f$ is in the interior of $B$. The picture on the left in Figure 4 shows the case $n=2$.

Note that $B=\left\{x \in \mathbb{R}^{n} \mid \sum_{i=1}^{n} x_{i} \leq n, x_{i} \geq 0, i=1, \ldots, n\right\}$. The point $e-e_{i}$, where $e$ denotes the vector of all ones, is the unique integral point in the relative interior of the facet 
of $B$ defined by $x_{i} \geq 0$ and $e$ is the unique integral point in the relative interior of the facet of $B$ defined by $\sum_{i=1}^{n} x_{i} \leq n$. Thus $\mathcal{I}=\left\{e, e-e_{1}, \ldots, e-e_{n}\right\}$.

Let $d^{1}, \ldots, d^{n+1}$ be defined as follows: $d^{i}=e_{i}-\frac{1}{n} f, i=1, \ldots, n$ and $d^{n+1}=-\frac{1}{n} f$. Then $R(e)=\left\{\sum_{j=1}^{n} \alpha_{j} d^{j} \mid 0 \leq \alpha_{i} \leq 1, i=1, \ldots, n\right\}$ and $R\left(e-e_{i}\right)=\left\{\sum_{j=1}^{n+1} \alpha_{j} d^{j} \mid 0 \leq \alpha_{k} \leq\right.$ $\left.1, k=1, \ldots, n+1, \alpha_{i}=0\right\}$. Therefore $R=\left\{\sum_{j=1}^{n+1} \alpha_{j} d^{j} \mid 0 \leq \alpha_{i} \leq 1, i=1, \ldots, n+1, \alpha_{i}=\right.$ 0 for some $i, 1 \leq i \leq n+1\}$

Lemma 11. Let $B=\operatorname{conv}\left\{0, n e_{1}, \ldots, n e_{n}\right\}$. For every $r \in \mathbb{R}^{n}$, there exists $w \in \mathbb{Z}^{n}$ such that $r+w \in R$.

Proof. Note that, for $1 \leq i, j \leq n+1, d^{i}-d^{j} \in \mathbb{Z}^{n}$.

Let $C_{i}=$ cone $\left\{d^{j} \mid j \neq i, 1 \leq j \leq n+1\right\}, i=1, \ldots, n+1$. Note that $\cup_{i=1}^{n+1} C_{i}=\mathbb{R}^{n}$ and $C_{i} \cap C_{k}=$ cone $\left\{d^{j} \mid j \neq i, k, 1 \leq j \leq n+1\right\}$. Furthermore, $-d^{i} \in C_{i}$ for $i=1, \ldots, n+1$.

Claim: Let $r \in \mathbb{R}^{n}$ and let $i$ such that $r \in C_{i}$. There exists a unique $\alpha \in \mathbb{R}^{n+1}$ such that $r=\sum_{j=1}^{n+1} \alpha_{j} d^{j}$ and $\alpha_{i}=0$. Furthermore, $\alpha$ is nonnegative and $\alpha_{j} \leq \alpha_{j}^{\prime}$ for every nonnegative $\alpha^{\prime} \in \mathbb{R}^{n+1}$ such that $r=\sum_{j=1}^{n+1} \alpha_{j}^{\prime} d^{j}$.

We prove the claim. Since $C_{i}$ is generated by $n$ linearly independent vectors, $r$ can be uniquely written as $r=\sum_{j=1}^{n+1} \alpha_{j} d^{j}$ such that $\alpha_{i}=0$, and $\alpha$ must be nonnegative since $r \in C_{i}$. Given a nonnegative $\alpha^{\prime} \in \mathbb{R}^{n+1}$ such that $r=\sum_{j=1}^{n+1} \alpha_{j}^{\prime} d^{j}$ distinct from $\alpha$, then $\alpha_{i}^{\prime}>0$. Hence

$$
-d^{i}=\left(\alpha_{i}^{\prime}\right)^{-1} \sum_{\substack{j=1 \\ j \neq i}}^{n+1}\left(\alpha_{j}^{\prime}-\alpha_{j}\right) d^{j}
$$

thus $\alpha_{j}^{\prime}-\alpha_{j} \geq 0$ since $-d^{i} \in C_{i}$, hence by the above argument $-d^{i}$ can be uniquely written as a linear combination of the extreme rays of $C_{i}$, and such combination is nonnegative. This proves the claim.

Let us now consider $r \in \mathbb{R}^{n}$. Let $i$ be such that $r \in C_{i}, 1 \leq i \leq n+1$. Let $\alpha \in \mathbb{R}^{n+1}$ such that $r=\sum_{j=1}^{n+1} \alpha_{j} d^{j}$ and $\alpha_{i}=0$. By the above claim $\alpha$ is nonnegative. Let $\bar{\alpha}=$ $\max _{j=1, \ldots, n+1} \alpha_{j}$. If $\bar{\alpha} \leq 1$, then $r \in R$. If not, $\alpha_{k}=\bar{\alpha}>1$ for some $1 \leq k \leq n+1$.

Let $r^{\prime}=r+\left(e_{i}-e_{k}\right)=r+\left(d^{i}-d^{k}\right)$. Then $r^{\prime}=\sum_{j \neq i, k} \alpha_{j} d^{j}+d^{i}+\left(\alpha_{k}-1\right) d^{k}$. Let $h$ be such that $r^{\prime} \in C_{h}, 1 \leq h \leq n+1$ and let $\alpha^{\prime} \in \mathbb{R}^{n+1}$ be the unique vector such that $r^{\prime}=\sum_{j=1}^{n+1} \alpha_{j}^{\prime} d^{j}$ and $\alpha_{h}^{\prime}=0$. By the previous claim, $\alpha^{\prime}$ satisfies the following properties

- $r^{\prime}-r \in \mathbb{Z}^{n}$ and $\alpha_{h}^{\prime}=0$,

. $0 \leq \alpha_{j}^{\prime} \leq \alpha_{j}, j \neq i, 1 \leq j \leq n+1$,

. $0 \leq \alpha_{i}^{\prime} \leq 1,0 \leq \alpha_{k}^{\prime} \leq \alpha_{k}-1$.

Thus, either $\max _{j=1, \ldots, n+1} \alpha_{j}^{\prime} \leq \bar{\alpha}-1$, or the number of indices $j$ such that $\alpha_{j}^{\prime}=\bar{\alpha}$ is smaller than the number of indices $j$ such that $\alpha_{j}=\bar{\alpha}$. This implies the statement of the lemma.

It can be shown that, in this case, $R$ is a polytope with $\left(\begin{array}{c}n+1 \\ 2\end{array}\right)$ pairs of parallel facets, and that $R$ has volume 1 . Thus, by Lemma 11, all possible translations of $R$ by integral vectors form a tiling of $\mathbb{R}^{n}$. Therefore for every $d \in \mathbb{R}^{n}$, there exists $w^{d} \in \mathbb{Z}^{n}$ such that $d+w^{d} \in R$. 
By Theorem 7, the function $\pi_{\ell}$ defined by $\pi_{\ell}(d)=\psi_{B}\left(d+w^{d}\right)$ is the unique minimal lifting of $\psi_{B}$.

Whenever $B$ is a maximal lattice-free simplex, $\psi_{B}$ is extreme for $(3)$. Indeed, if $v^{1}, \ldots, v^{n+1}$ are the vertices of $B$ and we define $r^{j}=v^{j}-f, j=1, \ldots, n+1, \psi_{B}$ is extreme for (3) if and only if $\sum_{j=1}^{n+1} \psi_{B}\left(r^{j}\right) s_{j} \geq 1$ is extreme for the convex hull of the set $R_{f}\left(r^{1}, \ldots, r^{n+1}\right)$ defined as the set of all $s \in \mathbb{R}^{n+1}$ such that $f+\sum_{j=1}^{n+1} r^{j} s_{j} \in \mathbb{Z}^{n}$ and $s \geq 0$ (see [11]). In this case, since each facet of $B$ contains an integral point, for $i=1, \ldots, n+1$ there exists $s^{i} \in \mathbb{R}^{n+1}$ such that $s_{j}^{i}>0$ for all $j \neq i, 1 \leq j \leq n+1, s_{i}^{i}=0$ and $\sum_{j=1}^{n+1} s_{j}^{i} r^{j} \in \mathbb{Z}^{n}$. Hence $s^{1}, \ldots, s^{n+1}$ are linearly independent points of $R_{f}\left(r^{1}, \ldots, r^{n+1}\right)$, and $\sum_{j=1}^{n+1} \psi_{B}\left(r^{j}\right) s_{j}^{i}=1$ for $i=1, \ldots, n+1$. This shows that $\sum_{j=1}^{n+1} \psi_{B}\left(r^{j}\right) s_{j} \geq 1$ defines a facet of $\operatorname{conv}\left(R_{f}\left(r^{1}, \ldots, r^{n+1}\right)\right)$, and thus it is extreme for $\operatorname{conv}\left(R_{f}\left(r^{1}, \ldots, r^{n+1}\right)\right)$. Therefore $\psi_{B}$ is extreme for (3).

The above statement and Remark 2 imply the following.

Theorem 12. If $B=\operatorname{conv}\left(0, n e_{1}, \ldots, n e_{n}\right),\left(\psi_{B}, \pi_{\ell}\right)$ is extreme for (1) with $S=\mathbb{Z}^{n}$.

The above theorem holds up to unimodular transformations and integer translations of the set $B$. We recall that an integral square matrix is unimodular if it has determinant \pm 1 . Given any unimodular $n \times n$-matrix $U$ and vector $v \in \mathbb{Z}^{n}$ such that $f$ is in the interior of the set $B^{\prime}=\operatorname{conv}\left(v, n\left(U e_{1}\right)+v, \ldots, n\left(U e_{n}\right)+v\right)$ (which is lattice-free by construction), then $\left(\psi_{B^{\prime}}, \pi_{\ell}\right)$ is extreme for (1) when $S=\mathbb{Z}^{n}$. Note that, given a vector $f \notin \mathbb{Z}^{n}$, one may always find an appropriate unimodular matrix $U$ and integral vector $v$ so that $f$ is in the interior of the corresponding set $B^{\prime}$.

Recent computational work has focused mostly on inequalities valid for two-row problems (i.e. $n=2$ ). The function $\psi_{B^{\prime}}$ and the corresponding minimal lifting provide inequalities for many rows, and can be computed efficiently. This type of lifted inequalities have been used in computational experiments by Espinoza [12] (using values of $n$ up to 10), and the results indicate that such cuts might be useful in practice.

\subsection{Simple cones}

We consider the case were $S=\mathbb{Z}^{n-1} \times \mathbb{Z}_{+}$and the maximal $S$-free convex set $B$ is the translation of a simple cone. That is, $B$ has a unique vertex $v$, and $B-v$ is a simple cone. Recall that a polyhedral cone in $\mathbb{R}^{n}$ is simple if it is generated by $n$ linearly independent vectors, and therefore it has $n$ facets. This case extends the wedge inequalities of Section 3.1.

Let $B=\left\{x \in \mathbb{R}^{n} \mid a_{i}(x-f) \leq 1, i=1 \ldots, n\right\}$. By Theorem $3, \operatorname{rec}(B) \cap \operatorname{rec}(\operatorname{conv}(S))$ is contained in the lineality space of $B$, which is empty. Therefore $B \cap \operatorname{conv}(S)$ is bounded. Therefore the polytope $B \cap\left(\mathbb{R}^{n-1} \times\{0\}\right)$ is an an $(n-1)$-dimensional simplex $P$. Let $v^{1}, \ldots, v^{n}$ be the vertices of $P$, and let $r^{j}=v^{j}-f, j=1, \ldots, n$. By symmetry, we may assume that $a_{i} r^{j}=1$ for $1 \leq i, j \leq n, i \neq j$, and $a_{i} r^{i}<1$. Let $\hat{r}=v-f$. Note that, for $i=1, \ldots, n$, $a_{i} \hat{r}=1$.

Let $\bar{x}$ be a point of $S$ in the relative interior of one of the facets of $B$, say the facet defined by $a_{h}(x-f) \leq 1$. Then $\bar{x}$ can be uniquely written as $\bar{x}=f+\bar{\alpha} \hat{r}+\sum_{j=1}^{n} \bar{\alpha}_{j} r^{j}$ such that $0 \leq \bar{\alpha}_{j}, j=1, \ldots, n$, and $\bar{\alpha}_{h}=0$. Let $R(\bar{x})=\left\{\sum_{j=1}^{n} \alpha_{j} r^{j} \mid 0 \leq \alpha_{j} \leq \bar{\alpha}_{j}, j=1, \ldots, n\right\}+\langle\hat{r}\rangle$. Let us denote by $\mathcal{I}$ the set of all points $\bar{x}$ in $S$ such that $\bar{x}$ is contained in the relative interior of some facet of $B$. Let $R=\cup_{\bar{x} \in \mathcal{I}} R(\bar{x})$. 
Lemma 13. For every $d \in R, \pi_{\ell}(d)=\psi_{B}(d)$.

Proof. We only need to show that, given $\bar{x} \in \mathcal{I}$ and $d \in R(\bar{x}), \pi_{\ell}(d)=\psi_{B}(d)$. By symmetry we may assume that $\bar{x}$ is in the relative interior of the facet defined by $a_{1}(x-f) \leq 1$. Let $\bar{\alpha} \in \mathbb{R}$ and $\bar{\alpha}_{2}, \ldots, \bar{\alpha}_{n}$ nonnegative such that $\bar{x}=f+\bar{\alpha} \hat{r}+\sum_{j=2}^{n} \bar{\alpha}_{j} r^{j}$. Since $d \in R(\bar{x})$, there exist $\alpha \in \mathbb{R}$ and $\alpha_{1}, \ldots, \alpha_{n}$ such that $d=\alpha \hat{r}+\sum_{j=2}^{n} \alpha_{j} r^{j}$ and $0 \leq \alpha_{j} \leq \bar{\alpha}_{j}, j=2, \ldots, n$.

Note that, for $i=2, \ldots, t,\left(a_{1}-a_{i}\right) d=\alpha\left(a_{1}-a_{i}\right) \hat{r}+\sum_{j=2}^{n} \alpha_{j}\left(a_{1}-a_{i}\right) r^{j} \geq 0$, since $\left(a_{1}-a_{i}\right) \hat{r}=0$ and $\left(a_{1}-a_{i}\right) r^{j} \geq 0$. Thus $\psi_{B}(d)=a_{1} d$.

We will show that, for every $\lambda<\psi_{B}(d)$, the set $B(\lambda)$ defined in (7) contains the point $\left(\begin{array}{l}\bar{x} \\ 1\end{array}\right)$ in its interior. By Theorem 4, this will imply $\pi_{\ell}(d) \geq \psi_{B}(d)$, and thus $\pi_{\ell}(d)=\psi_{B}(d)$.

Let $\lambda=\psi_{B}(d)-\delta$ for some $\delta>0$. Then $B(\lambda)$ is the set of $x \in \mathbb{R}^{n+1}$ satisfying

$$
\begin{aligned}
& a_{1}(x-f) \quad-\delta x_{n+1} \leq 1, \\
& a_{i}(x-f)+\left(a_{1}-a_{i}\right) d x_{n+1}-\delta x_{n+1} \leq 1 \quad i=2, \ldots, t .
\end{aligned}
$$

Substituting $\left(\begin{array}{l}\bar{x} \\ 1\end{array}\right)$ in the first inequality, we obtain $a_{1}(\bar{x}-f)-\delta=1-\delta<1$. Substituting in the $i$ th inequality, $i=2, \ldots, n+1$, we obtain

$$
\begin{aligned}
a_{i}(\bar{x}-f)+\left(a_{1}-a_{i}\right) d-\delta & =\bar{\alpha} a_{i} \hat{r}+\sum_{j=2}^{n} \bar{\alpha}_{j} a_{i} r^{j}+\alpha\left(a_{1}-a_{i}\right) \hat{r}+\sum_{j=2}^{n} \alpha_{j}\left(a_{1}-a_{i}\right) r^{j}-\delta \\
& =\bar{\alpha} a_{1} \hat{r}+\sum_{j=2}^{n} \bar{\alpha}_{j} a_{1} r^{j}-\bar{\alpha}_{i}\left(a_{1}-a_{i}\right) r^{i}+\alpha_{i}\left(a_{1}-a_{i}\right) r^{i}-\delta \\
& =a_{1}(\bar{x}-f)-\left(\bar{\alpha}_{i}-\alpha_{i}\right)\left(a_{1}-a_{i}\right) r^{i}-\delta \\
& \leq 1-\delta<1
\end{aligned}
$$

where the equality in the second line follows from $a_{i} \hat{r}=a_{1} \hat{r}$ and $a_{1} r^{j}=a_{i} r^{j}$ for all $2 \leq j \leq n$ such that $i \neq j$, while the first inequality on the last line follows from $\alpha_{i} \leq \bar{\alpha}_{i}$ and $a_{i} r^{i}<1=$ $a_{1} r^{i}$.

Note that $P$ is an $n$-1-dimensional simplex in $\mathbb{R}^{n-1} \times\{0\}$ and $P$ does not contain any point of $\mathbb{Z}^{n-1} \times\{0\}$ in its interior. Suppose that $P$ is maximal lattice free in $\mathbb{R}^{n-1} \times\{0\}$. In this case we can apply the results of Section 3.2 to identify cases where $\pi_{\ell}$ is a lifting of $\psi_{B}$.

Let $\bar{f}$ be the intersection of the line $f+\langle\hat{r}\rangle$ with $\mathbb{R}^{n-1} \times\{0\}$, and let $\bar{r}^{j}=v^{j}-\bar{f}$. For every point $\bar{x} \in \mathbb{Z}^{n-1} \times\{0\}$ in the relative interior of one of the facets of $P$, say the facet defined by $a_{h}(x-f) \leq 1, \bar{x}$ can be uniquely written as $\bar{x}=\bar{f}+\sum_{j=1}^{n} \bar{\alpha}_{j} \bar{r}^{j}$ such that $0 \leq \bar{\alpha}_{j}$, $j=1, \ldots, n$, and $\bar{\alpha}_{h}=0$. Let $\bar{R}(\bar{x})=\left\{\sum_{j=1}^{n} \alpha_{j} \bar{r}^{j} \mid 0 \leq \alpha_{j} \leq \bar{\alpha}_{j}, j=1, \ldots, n\right\}$. Note that $\bar{R}(\bar{x})=R(\bar{x}) \cap\left(\mathbb{R}^{n-1} \times\{0\}\right)$. Let $\overline{\mathcal{I}}$ be the set of all points in $\bar{x} \in \mathbb{Z}^{n-1} \times\{0\}$ in the relative interior of some of the facets of $P$. We define $\bar{R}=\cup_{\bar{x} \in \overline{\mathcal{I}}} \bar{R}(\bar{x})$. Then $R \supseteq \bar{R}+\langle\hat{r}\rangle$. Hence, if for every $r \in \mathbb{R}^{n-1} \times\{0\}$ there exists $w \in \mathbb{Z}^{n-1} \times\{0\}$ such that $r+w \in \bar{R}$, it also holds that for every $r \in \mathbb{R}^{n}$ there exists $w^{r} \in \mathbb{Z}^{n-1} \times\{0\}$ such that $r+w^{r} \in R$.

Since $\mathbb{R}^{n-1} \times\{0\}$ is the lineality space of $\operatorname{conv}(S)$, Theorem 7 implies that $\pi_{\ell}$ is the unique minimal lifting of $\psi_{B}$, and $\pi_{\ell}(r)=\psi\left(r+w^{r}\right)$.

The above property holds, for example, when $n=2$ and $P$ is an interval of length one (as seen in Section 3.1), when $n=3$ and $P$ is a maximal lattice-free triangle containing at least four points in $\mathbb{Z}^{2} \times\{0\}$, or for general $n$ when $P$ is a unimodular transformation of $\operatorname{conv}\left(0,(n-1) e_{1}, \ldots,(n-1) e_{n-1}\right)$. 


\section{References}

[1] K. Andersen, Q. Louveaux, R. Weismantel, L. A. Wolsey, Cutting Planes from Two Rows of a Simplex Tableau, Proceedings of IPCO XII, Ithaca, New York (June 2007), Lecture Notes in Computer Science 4513, 1-15.

[2] K. Andersen, Q. Louveaux, R. Weismantel, An Analysis of Mixed Integer Linear Sets Based on Lattice Point Free Convex Sets, manuscript, 2007.

[3] E. Balas, R.G. Jeroslow, Strengthening cuts for mixed integer programs, European Journal of Operations Research 4 (1980) 224-234.

[4] A. Basu, M. Conforti, G. Cornuéjols, G. Zambelli, A counterexample to a conjecture of Gomory and Johnson, manuscript (November 2008).

[5] A. Basu, M. Conforti, G. Cornuéjols, G. Zambelli, Maximal lattice-free convex sets in linear subspaces, manuscript (March 2009).

[6] A. Basu, M. Conforti, G. Cornuéjols, G. Zambelli, Minimal inequalities for an infinite relaxation of integer programs, manuscript (April 2009).

[7] A. Basu, G. Cornuéjols, G. Zambelli, Convex Sets and Minimal Sublinear Functions, manuscript (March 2009).

[8] V. Borozan, G. Cornuéjols, Minimal Valid Inequalities for Integer Constraints, technical report (July 2007), to appear in Mathematics of Operations Research.

[9] G. Cornuéjols, F. Margot, On the Facets of Mixed Integer Programs with Two Integer Variables and Two Constraints (September 2007), to appear in Mathematical Programming.

[10] S.S. Dey, L.A. Wolsey, Lifting Integer Variables in Minimal Inequalities Corresponding to Lattice-Free Triangles, IPCO 2008, Bertinoro, Italy (May 2008), Lecture Notes in Computer Science 5035, 463-475.

[11] S.S. Dey, L.A. Wolsey, Constrained Infinite Group Relaxations of MIPs, manuscript (March 2009).

[12] D. Espinoza, Computing with multi-row Gomory Cuts, Operations Research Letters (2009), to appear.

[13] R.E. Gomory, Some Polyhedra Related to Combinatorial Problems, Linear Algebra and Its Applications 2 (1969) 451-558.

[14] Z. Gu, G.L. Nemhauser, M.W.P. Savelsbergh, Sequence independent lifting in mixed integer programming, Journal of Combinatorial Optimization 4 (2000) 109-129.

[15] L. Lovász, Geometry of Numbers and Integer Programming, Mathematical Programming: Recent Developements and Applications, M. Iri and K. Tanabe eds., Kluwer (1989), 177-210. 
[16] M. Padberg, On the facial structure of set packing polyhedra, Mathematical Programming 5 (1973) 199-215.

[17] L.A. Wolsey, Valid inequalities and superadditivity for 0-1 integer programs, Mthematics of Operations Research 2 (1977) 66-77. 